\title{
Receivers for the uplink of multiuser Generalized Spatial Modulation MIMO systems
}

\author{
José Calpa, João Cal-Braz, Rodrigo Pereira David, and Raimundo Sampaio-Neto
}

\begin{abstract}
Generalized spatial modulation is envisioned as a promising communication paradigm for the next communication systems, in which high data rates and reliability are important features, but also energy and infrastructure-saving solutions are sought. This work presents a variety of efficient suboptimal signal detection strategies for the uplink of multiuser GSM MIMO systems. They are comprised of user decoupling strategies followed by GSM signal detection schemes, and offer different balances of detection performance and computational complexity. Simulation results exhibit the performance of these strategies under different system scenarios and channel propagation effects.
\end{abstract}

Index Terms-Multiuser MIMO, spatial modulation, singularvalue decomposition

\section{INTRODUCTION}

$\mathbf{I}$ $\mathrm{N}$ the next cellular communication system technologies, low-cost infrastructure and massive antenna devices are key features. In this scenario, spatial modulation (SM)-based communication systems are envisioned as promising techniques due to: 1- the possibility of a striking reduction of the number of RF chains required at the transmitter, and 2- the potential spectral efficiency increase. SM is a multiple-input multipleoutput (MIMO) system that, at each transmission slot, the information is conveyed by the transmission of a symbol by a single antenna, and also by the index associated to the antenna that emitted this symbol [1]. Specifically, Generalized Spatial Modulation (GSM) is of particular interest due to the attractive balance of the spectral efficiency distributed between conventional modulated symbols and transmit antenna combinations [2], as in this system a subset of the antennas available at the transmitter is active simultaneously.

Optimal and suboptimal detection schemes have been proposed for the point-to-point SM/GSM MIMO context [2][5]. When the uplink communication of a multiuser system is considered, significant developments focus on single-activeantenna SM, such as [6], that presented the optimal detector in this scenario, and [7], that proposes a hybrid-processing-based approach for the SM uplink system in millimeter wave communication. To the best of our knowledge, [8], that proposes a near-optimal strategy, relies on the channel-hardening effect, suitable only in large-scale system, is the only relevant work for the uplink of GSM communication.

José Calpa and Raimundo Sampaio-Neto are with the Pontifical Catholic University of Rio de Janeiro, RJ, 22451-900, Brazil. E-mail: \{joselocalpa14, raimundo\}@cetuc.puc-rio.br

João Cal-Braz and Rodrigo David are with the National Institute of Metrology, Quality and Technology, Duque de Caxias, RJ, 25250-020, Brazil. E-mail: \{jabraz, rpdavid\}@inmetro.gov.br

Digital Object Identifier: 10.14209/jcis.2020.14
Given the scarcity of literature devoted to the uplink problem of GSM systems in a regular multiuser MIMO scenario, and, moreover, as optimal detection is infeasible due to prohibitive computational cost, this work presents several suboptimal receivers to be applied at the base station (BS) to retrieve the information sent by the users, and extends the work presented in [9]. The strategies presented herein are divided into two categories: a- when the BS jointly processes the data vector sent by all users at once, and b- when the BS first decouples the data belonging to different users and the applies detection techniques to retrieve the data vector of each user separately. Thus, this receiver category is comprised of the pair decoupler/detector. The use of decouplers in the downlink of multiuser communication is mandatory, as a user needs to extract its information from the interference destined to other users, so decoupling strategies are used in precoders at the BS [10]. This paper adapts these strategies done by precoders into equalizers in uplink communication, aiming at achieving computational complexity savings compared to the joint detection by the BS of the information coming from all users. The trade-off between detection performance and computational complexity is analyzed. Furthermore, the equivalence between two receiver strategies, namely, D-SVD/ML-W and D-ZF/ML$\mathrm{C}$ is analytically demonstrated.

Notation: Lower and upper boldface symbols denote column vectors and matrices, respectively. $\|\mathbf{x}\|$ represents the two-norm of a vector $\mathbf{x} .(\cdot)^{T},(\cdot)^{\mathcal{H}},(\cdot)^{\dagger}$ are used to denote transpose, Hermitian transpose, and left pseudoinverse of their argument, respectively. $\mathbf{0}_{n \times m}$ corresponds to the matrix of size $n \times m$ with all entries equal to zero. $\mathbb{E}[\cdot],(\cdot)$, and $\lfloor\cdot\rfloor$ correspond to the expectation, to the binomial coefficient and to the floor operation, respectively.

\section{MU-GSM-MIMO SYSTEM MODEL}

Consider the uplink of a multiuser generalized spatial modulation MIMO (MU-GSM-MIMO) system, in which $K$ users transmit data to a BS with $N_{R}$ antenna elements. During a timeslot, each user, equipped with $N_{T}$ transmit antennas, performs GSM data transmission. In this scheme, $N_{A}\left(N_{A}<N_{T}\right)$ transmit antennas are activated, and through them independent QAM symbols, belonging to an $M$-length constellation $\mathbb{B}$, are transmitted. The choice of the active antennas is commanded by the data to be transmitted by this user, as defined in an antenna combination mapping table (ACMT) and collected in the set $\mathbb{I}$. For instance, consider a user equipped with $N_{T}=4$ and $N_{A}=2$. Under this configuration, $N_{C}$ is the number of permissible antenna configurations, $l_{1}, l_{2}, \ldots l_{N_{C}} \in \mathbb{I}$, equals 
TABLE I

EXAMPLE OF AN ACMT MAPPING TABLE FOR $N_{T}=4$ AND $N_{A}=2$. (ENTRIES 1 AND 0 REPRESENT ACTIVE AND NON-ACTIVE ANTENNAS, RESPECTIVELY.)

\begin{tabular}{|c|c|c|c|c|}
\hline Input Bits & Antenna 1 & Antenna 2 & Antenna 3 & Antenna 4 \\
\hline 00 & 1 & 1 & 0 & 0 \\
\hline 01 & 1 & 0 & 1 & 0 \\
\hline 10 & 0 & 1 & 0 & 1 \\
\hline 11 & 0 & 0 & 1 & 1 \\
\hline
\end{tabular}

the power of two immediately lower than $\left(\begin{array}{l}N_{T} \\ N_{A}\end{array}\right)$, or equal to 4 , in this example. So, input binary data are mapped into antenna combinations, as exemplified in the ACMT presented in Table I. As a result, the number of bits transmitted per timeslot, $\eta$, equals the sum of the bits given by the antenna combination and the bits associated to the QAM symbols transmitted by the active antennas $\eta=\left\lfloor\log _{2}\left(\begin{array}{l}N_{T} \\ N_{A}\end{array}\right)\right\rfloor+N_{A} \log _{2} M$. The same number of transmit antennas at each user is considered, as well as the number of active antennas. Moreover, a unique ACMT shared by all users, known by the BS, is admitted. The strategies developed herein are easily extended to the general case, where different number of antennas or different ACMT are associated to each user.

Let $\mathbf{b}_{n} \in \mathbb{B}^{N_{A}}$ be the vector containing equiprobable modulated symbols, $b_{i, n}, i=1, \ldots, N_{A}$, transmitted by the $n$th user, with average energy $\mathbb{E}\left[b_{i, n}^{*} b_{i, n}\right]=E_{s}$. Consider the set $\mathbb{B}_{0}=\{0\} \cup \mathbb{B}$. The information vector transmitted by the $n$th user is $\mathbf{s}_{n}^{l_{m}} \in \mathbb{B}_{0}^{N_{T}}$, which contains the symbols in $\mathbf{b}_{n}$ in the entries related to the active antennas specified by the antenna combination $l_{m}$, and zeroes elsewhere. For the sake of simplicity of notation, the information vector is represented by $\mathbf{s}_{n}$ onwards. The signals from the users traverses the MIMO channel and are collected by the $N_{R}$ receive antenna elements at the BS, as given by $\mathbf{y} \in \mathbb{C}^{N_{R}}$ :

$$
\mathbf{y}=\sum_{n=1}^{K} \mathbf{H}_{n} \mathbf{s}_{n}+\mathbf{n}
$$

where $\mathbf{H}_{n} \in \mathbb{C}^{N_{R} \times N_{T}}$ is the channel matrix that links the $N_{T}$ antennas of the $n$th user to the BS, with elements $h_{i j}$ such that $\mathbb{E}\left[h_{i j}^{*} h_{i j}\right]=1$. The additive Gaussian noise, represented by $\mathbf{n}$, is zero-mean and its covariance matrix is equal to $\mathbf{K}_{n}=\sigma_{n}^{2} \mathbf{I}_{N_{R}}$. Epression (1) can be rewritten as $\mathbf{y}=\mathbf{H s}+\mathbf{n}$, where $\mathbf{H}=\left[\begin{array}{llll}\mathbf{H}_{1} & \mathbf{H}_{2} & \ldots & \mathbf{H}_{K}\end{array}\right]$ and $\mathbf{s}=\left[\begin{array}{lll}\mathbf{s}_{1}^{T} & \mathbf{s}_{2}^{T} & \ldots \\ \mathbf{s}_{K}^{T}\end{array}\right]^{T}$ with dimensions $N_{R} \times K N_{T}$ and $K N_{T} \times 1$, respectively. The perfect knowledge of the channel $\mathbf{H}$ at the BS is assumed.

\section{RECEIVERS}

In this section, receivers for MU-GSM-MIMO systems are proposed. These receivers are comprised of decouplers, that remove the interference of the other users over the intended user, followed by detectors of the transmitted SM data vector.

\section{A. User Decoupling}

User decoupling techniques aim at the removal of the interference produced by the other users, and the ones presented here are based on the projection of the signal $\mathbf{y}$ on the null subspace of these interferences. All three presented strategies are based on the determination of the matrix $\mathbf{A}_{n} \in \mathbb{C}^{N_{T} \times N_{R}}$, belonging in the left nullspace of $\tilde{\mathbf{H}}_{n}$, or $\mathbf{A}_{n} \tilde{\mathbf{H}}_{n}=\mathbf{0}, \quad n \in$ $\{1,2, \ldots, K\}$. Here $\tilde{\mathbf{H}}_{n} \in \mathbb{C}^{N_{R} \times(K-1) N_{T}}$ is built excluding from $\mathbf{H}$ the columns that refer to user $n$, then: $\tilde{\mathbf{H}}_{n}=$ $\left[\begin{array}{llllll}\mathbf{H}_{1} & \ldots & \mathbf{H}_{n-1} & \mathbf{H}_{n+1} & \ldots & \mathbf{H}_{K}\end{array}\right]$

1) Projection in the nullspace of the interferences by singular value decomposition $(D-S V D)$ : This strategy uses singular value decomposition (SVD) of the channel matrix $\tilde{\mathbf{H}}_{n}$, which contains interference components, i.e., $\tilde{\mathbf{H}}_{n}=\tilde{\mathbf{U}}_{n} \tilde{\boldsymbol{\Sigma}}_{n} \tilde{\mathbf{V}}_{n}^{\mathcal{H}}$, where $\tilde{\mathbf{U}}_{n}$ and $\tilde{\mathbf{V}}_{n}^{\mathcal{H}}$ are matrices comprised of singular vectors and $\tilde{\boldsymbol{\Sigma}}_{n}$ is a matrix of singular values. Let $r_{n}$ be the rank of $\tilde{\mathbf{H}}_{n}, r_{n}=\operatorname{rank}\left(\tilde{\mathbf{H}}_{n}\right) \leq(K-1) N_{T}$, then the SVD is expressed by :

$$
\tilde{\mathbf{H}}_{n}=\left[\begin{array}{ll}
\tilde{\mathbf{U}}_{1, n} & \tilde{\mathbf{U}}_{0, n}
\end{array}\right] \tilde{\boldsymbol{\Sigma}}_{n}\left[\begin{array}{ll}
\tilde{\mathbf{V}}_{1, n} & \tilde{\mathbf{V}}_{0, n}
\end{array}\right]^{\mathcal{H}}
$$

where $\tilde{\mathbf{U}}_{0, n}$ and $\tilde{\mathbf{V}}_{0, n}^{\mathcal{H}}$ with dimensions $N_{R} \times\left(N_{R}-r_{n}\right)$ and $\left((K-1) N_{T}-r_{n}\right) \times\left((K-1) N_{T}\right)$ compose orthogonal bases of the left nullspace and the right nullspace of $\tilde{\mathbf{H}}_{n}$ respectively. Then, in this strategy, the matrix $\mathbf{A}_{n}=\mathbf{A}_{n}^{\mathrm{SVD}}$ is given by $\mathbf{A}_{n}^{\mathrm{SVD}}=\tilde{\mathbf{U}}_{0, n}^{\mathcal{H}}$.

2) Projection in the nullspace of the interferences by zeroforcing filtering $(D-Z F)$ : This strategy is based on the channel inversion, and uses the zero-forcing (ZF) of the full channel matrix to perform the interference cancellation. Let $\mathbf{H}^{\dagger}=\left(\mathbf{H}^{\mathcal{H}} \mathbf{H}\right)^{-1} \mathbf{H}^{\mathcal{H}}$, of dimension $K N_{T} \times N_{R}$, be the $\mathrm{ZF}$ matrix of $\mathbf{H}$ that produces $\mathbf{H}^{\dagger} \mathbf{H}=\mathbf{I}$. Thus, $\mathbf{H}^{\dagger}$ is comprised of the submatrices $\mathbf{H}_{k}^{z}, k=1,2, \ldots, K$, such that $\mathbf{H}^{\dagger}=\left[\begin{array}{llll}\left(\mathbf{H}_{1}^{z}\right)^{T} & \left(\mathbf{H}_{2}^{z}\right)^{T} & \ldots & \left(\mathbf{H}_{K}^{z}\right)^{T}\end{array}\right]^{T}$. Then, the matrix, $\mathbf{A}_{n}=\mathbf{A}_{n}^{\mathrm{ZF}}$, that decouples the $n$th class from the others is $\mathbf{A}_{n}^{\mathrm{ZF}}=\mathbf{H}_{n}^{z}$.

3) Projection in the nullspace of the interferences using MMSE filtering (D-MMSE): The D-MMSE decoupler proposed in [11], is given by $\mathbf{F}^{M M S E}=\mathbf{H}^{\mathcal{H}}\left(\mathbf{H H}^{\mathcal{H}}+\right.$ $\left.\sigma_{n}^{2} \mathbf{I}\right)^{-1}$. Likewise D-ZF, $\mathbf{F}^{M M S E}$ is structured as $\mathbf{F}^{M M S E}=$ $\left[\begin{array}{llll}\left(\mathbf{F}_{1}\right)^{T} & \left(\mathbf{F}_{2}\right)^{T} & \ldots & \left(\mathbf{F}_{K}\right)^{T}\end{array}\right]$. In fact, $\mathbf{F}_{n}$ is approximately in the left nullspace of $\tilde{\mathbf{H}}_{n}$. In this procedure, the bases of the vector space generated by $\mathbf{F}_{n}$ are obtained by the $\mathbf{R Q}$ decomposition of $\mathbf{F}_{n}$, this means

$$
\mathbf{F}_{n}=\mathbf{R}_{n} \mathbf{Q}_{n}, \quad \forall n \in(1,2, \ldots, K),
$$

where $\mathbf{R}_{n}$ and $\mathbf{Q}_{n}$, have dimensions $N_{T} \times N_{T}$ and $N_{T} \times N_{R}$, are an upper triangular matrix and an orthonormal lines matrix which form the bases of the space $\mathbf{F}_{n}$, respectively. Then, the matrix, $\mathbf{A}_{n}=\mathbf{A}_{n}^{\mathrm{MMSE}}$, that separates the $n$th user out of the other ones is given by $\mathbf{A}_{n}^{\mathrm{MMSE}}=\mathbf{Q}_{n}$.

For the three strategies presented above, the decouplers act on the received signal in the BS, as given by $\tilde{\mathbf{y}}=\mathbf{A}_{n} \mathbf{y}$.

In the D-SVD decoupler, $\mathbf{A}_{n}^{\text {SVD }}$ is exactly in the left nullspace of the interferences. Then, can be written as:

$$
\tilde{\mathbf{y}}_{n}=\mathbf{A}_{n}^{\mathrm{SVD}} \mathbf{H}_{n} \mathbf{s}_{n}+\mathbf{A}_{n}^{\mathrm{SVD}} \mathbf{n}=\mathbf{U}_{0, n}^{\mathcal{H}} \mathbf{H}_{n} \mathbf{s}_{n}+\mathbf{U}_{0, n}^{\mathcal{H}} \mathbf{n},
$$

where $\tilde{\mathbf{y}}_{n} \in \mathbb{C}^{N_{T} \times 1}$ and the noise component remains white with covariance matrix $\mathbf{K}_{n}=\sigma_{n}^{2} \mathbf{I}_{N_{T}}$. For the strategy D$\mathrm{ZF}$, the application of the filter $\mathbf{A}_{n}^{\mathrm{ZF}}$, which eliminates the interferences on the $n$th user, processes $\mathbf{y}$, as given by:

$$
\tilde{\mathbf{y}}_{n}=\mathbf{A}_{n}^{\mathrm{ZF}} \mathbf{H}_{n} \mathbf{s}_{n}+\mathbf{A}_{n}^{\mathrm{ZF}} \mathbf{n}=\mathbf{s}_{n}+\mathbf{H}_{n}^{z} \mathbf{n}=\mathbf{s}_{n}+\mathbf{n}_{n},
$$


where the colored noise component $\mathbf{n}_{n}=\mathbf{H}_{n}^{z} \mathbf{n}$ with covariance matrix $\mathbf{K}_{\mathbf{n}_{\mathbf{n}}}=\sigma_{n}^{2}\left(\mathbf{H}_{n}^{\mathcal{H}} \mathbf{H}_{n}\right)^{-1}$. The matrix $\mathbf{K}_{n_{n}}$ is identified as the $n$th submatrix of dimension $N_{T} \times N_{T}$ on the main diagonal of matrix $\mathbf{K}_{n}=\sigma_{n}^{2}\left(\mathbf{H}^{\mathcal{H}} \mathbf{H}\right)^{-1}$.

In the strategy D-MMSE, $\mathbf{A}_{n}^{\mathrm{MMSE}}$, is approximately in the left nullspace of the interferences. Assuming that $\mathbf{A}_{n}^{\mathrm{MMSE}} \sum_{m=1, m \neq n}^{N} \mathbf{H}_{m} \mathbf{s}_{m} \approx \mathbf{0}_{N_{T} \times 1}$ in the expression, we obtain:

$$
\tilde{\mathbf{y}}_{n}=\mathbf{A}_{n}^{\mathrm{MMSE}} \mathbf{H}_{n} \mathbf{s}_{n}+\mathbf{A}_{n}^{\mathrm{MMSE}} \mathbf{n}=\mathbf{Q}_{n} \mathbf{H}_{n} \mathbf{s}_{n}+\mathbf{Q}_{n} \mathbf{n},
$$

where the noise component remains white with covariance matrix $\mathbf{K}_{n}=\sigma_{n}^{2} \mathbf{I}_{N_{T}}$.

\section{B. Detectors}

After the separation of the intended user, the information vector is retrieved using adequate detection strategy. Here, GSM detectors, with optimal and suboptimal performance, are presented.

1) Maximum likelihood detector in colored noise (ML-C): As the elements of $\mathbf{B}_{0}$ are equiprobable, the optimal detector of the $n$th user in the presence of colored noise is given by

$$
\hat{\mathbf{s}}_{n}=\underset{\substack{\mathbf{s}_{n} \in \mathbb{B}_{0} \\ l_{m} \in \mathbb{I}}}{\operatorname{argmin}}\left(\tilde{\mathbf{y}}_{n}-\mathbf{A}_{n} \mathbf{H}_{n} \mathbf{s}_{n}^{l_{m}}\right)^{\mathcal{H}} \mathbf{K}_{n_{n}}^{-1}\left(\tilde{\mathbf{y}}_{n}-\mathbf{A}_{n} \mathbf{H}_{n} \mathbf{s}_{n}^{l_{m}}\right)
$$

2) Maximum likelihood detector in white noise $(M L-W)$ : If the entries of the additive noise vector are uncorrelated, the detector presented in (7) is simplified to:

$$
\hat{\mathbf{s}}_{n}=\underset{\substack{\mathbf{s}_{n} \in \mathbb{B}_{0} \\ l_{m} \in \mathbb{I}}}{\operatorname{argmin}}\left\|\tilde{\mathbf{y}}_{n}-\mathbf{A}_{n} \mathbf{H}_{n} \mathbf{s}_{n}^{l_{m}}\right\|^{2}
$$

3) Projection-Based List Detector (PBLD): This suboptimal detector, proposed in [5], performs separately the detection of the transmit antenna combination and the symbol vector, implementing a list-detection scheme that generates candidates to the GSM information vector of the $n$th user. In the first stage, the BS applies a filter bank on the received vector. Each element of this bank projects the received data vector on the subspace generated by the channel matrix associated to the transmit antenna combination employed in the current transmission. Then, supposing that the channel matrix associated to a transmit antenna combination labeled as $q$ is denoted by $\mathbf{H}_{(q)} \in \mathbb{C}^{N_{R} \times N_{A}}$, which is a submatrix of $\mathbf{H}_{n}$, then the corresponding projection matrix belonging to the BS bank, $\mathbf{W}_{q} \in \mathbf{C}^{N_{R} \times N_{R}}$, is expressed by :

$$
\mathbf{W}_{q}=\mathbf{H}_{(q)}\left(\mathbf{H}_{(q)}^{\mathcal{H}} \mathbf{H}_{(q)}\right)^{-1} \mathbf{H}_{(q)}^{\mathcal{H}}, \quad q=1,2, \ldots, N_{C} .
$$

Hence, the filter banks totals $N_{C}$ projection matrices. In the strategy, the filter bank is responsible for sorting the most likely transmit antenna combinations used by user $n$. So, the Phase 1 sorts the combinations of transmitting antennas in decreasing order of the projection magnitude.

$$
\left\{\begin{array}{llll}
o_{1} & o_{2} & \ldots & o_{N_{c}}
\end{array}\right\}=\underset{q}{\operatorname{argsort}}\left\|\mathbf{W}_{q} \mathbf{y}\right\| .
$$

As a result $\left\|\mathbf{W}_{o_{1}} \mathbf{y}\right\| \geq\left\|\mathbf{W}_{o_{2}} \mathbf{y}\right\| \geq \cdots \geq\left\|\mathbf{W}_{o_{N_{c}}} \mathbf{y}\right\|$. The antenna combinations ordered in (10) are serially passed, starting from $o_{1}$ toward the smaller magnitude combinations, by the detector composed by a $\mathrm{ZF}$ equalizer $\mathbf{G}_{o_{i}}=\mathbf{H}_{\left(o_{i}\right)}^{\dagger}$ followed by an elementwise discretizer to produce the candidate, $\mathbf{c}_{o_{i}}=\mathcal{D}\left(\mathbf{G}_{o_{i}} \mathbf{y}\right)$, of the detected GSM information symbol of user $n$, being $\mathcal{D}$ the function that discretizes the input vector into the symbols of the employed QAM modulation. To this candidate is determined the Euclidean distance in relation to the data vector, producing $\epsilon_{o_{i}}:=\left\|\mathbf{y}-\mathbf{H}_{o_{i}} \mathbf{c}_{o_{i}}\right\|$

This is used as the metric of quality of the candidate relative to the received data, $\mathbf{y}$, and also as the input of the scheme that defines the list size $\lambda=\eta\left(\epsilon_{o_{i}}\right)$, represented here as the function $\eta$ :

Details about the operation of the dynamic control of the list size are found in [5]. The expressions for $\mathbf{c}_{o_{i}}, \epsilon_{o_{i}}$ and $\lambda$ are sequentially repeated until that the number of processed candidates is equal or higher than the list size. Then the candidate with the smallest Euclidean distance is selected as the detected symbols vector, $\hat{\mathbf{s}}$.

4) Projection-Based List Detector with lattice reduction (PBLD-LR): The PBLD-LR [5] detector differs from the PBLD due to the lattice reduction scheme, which reduces the loss of performance of the ZF filter in the occasions when the channel matrix is close to singular. In this scheme, the candidate expression, is modified to $\mathbf{c}_{o_{i}}=\mathcal{D}\left(\mathbf{T}_{o_{i}} \mathcal{Q}_{L R}\left(\mathbf{G}_{o_{i}} \mathbf{y}\right)\right)$, where $\mathbf{G}_{o_{i}}^{L R}$ is the $\mathrm{ZF}$ filter modified for the lattice reduction, $\mathcal{Q}_{L R}$ represents the quantization in a lattice reduction domain and $\mathbf{T}_{o_{i}}$ the matrix that transform the channel matrix into a nearly orthogonal matrix. Details about this process are found in [12].

\section{RESUlts}

The elements of the channel matrix $\mathbf{H}$ are modeled as statistically independent circularly symmetric complex Gaussian random variables, all with zero mean and unit variance. In all simulations, users are equipped with $N_{T}=4$ and $N_{A}=2$ and transmitting QPSK modulated symbols. The ratio between the signal component and the noise component in (1), experienced at each BS receive antenna is defined as the signal-to-noise ratio $(\mathrm{SNR})$ and is given by $\mathrm{SNR}=10 \log _{10}\left(K N_{T} E_{s} / \sigma_{n}^{2}\right)$.

Figs. 1 and 2 show the detection performance of the receiving algorithms, considering 4 users and 16 users, respectively. In the case of 4 users, comparison with strategies that perform the joint detection of all users at the BS (i.e. no user decoupling phase), by means of the suboptimal PBLD and PBLD-LR used to detect the vector $\mathbf{s}$ at once, was also included. Due to the prohibitive computational complexity, these joint detectors were not implement in the 64-user case.

In terms of detection performance, in both 4 and 16 users case, receivers based on D-MMMSE decouplers exhibit a performance superiority over those ZF- and SVD-based decouplers. In addition, a slight performance degradation is observed when the suboptimal detection strategy based on PBLD-LR is used instead of the optimal ML-W and ML-C.

Here, the identical performance of the strategies $\mathrm{D}-\mathrm{SVD} / \mathrm{ML}-\mathrm{W}$ and $\mathrm{D}-\mathrm{ZF} / \mathrm{ML}-\mathrm{C}$ is noteworthy. In fact, 


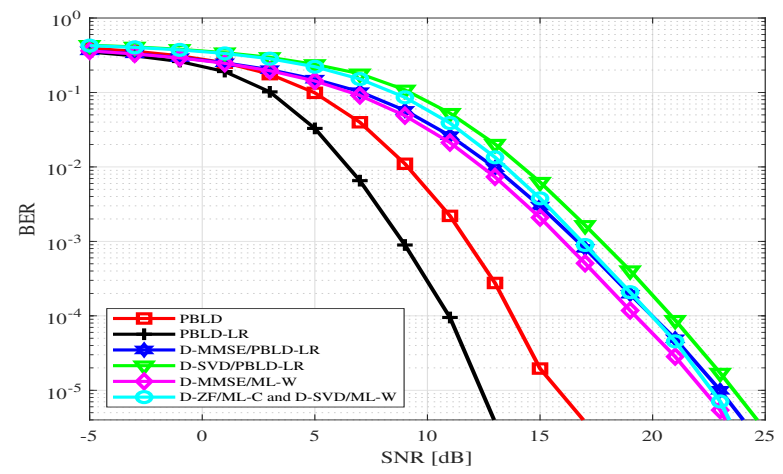

Fig. 1. Bit error rate of the receivers -4 users, $N_{R}=16$.

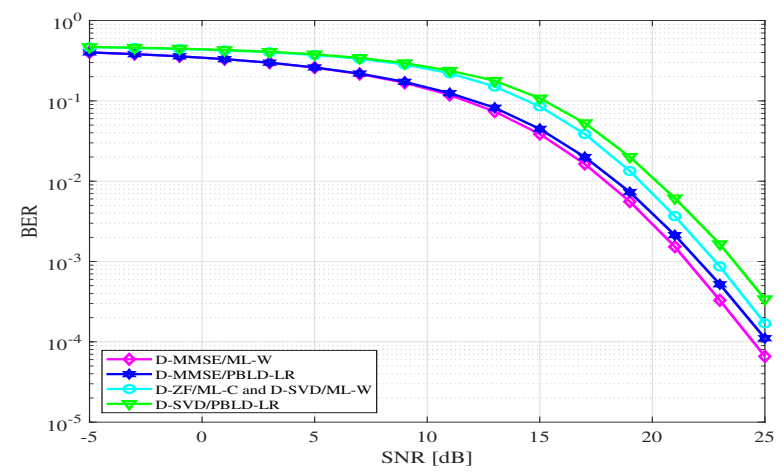

Fig. 2. Bit error rate of the receivers -16 users, $N_{R}=64$.

these strategies, although presenting distinct computational complexities, are equivalent as can be formally proved [13].

Computational complexity results are shown in Table II. In all configurations used in this table, $N_{R}=K N_{T}$. In configurations $\mathrm{A}, \mathrm{B}$ and $\mathrm{C}$ the number of users is set to 4 , 8 , and 16 , respectively.

Although the joint detection strategies PBLD and PBLDLR exhibit a large performance advantage compared to the receivers that employ the decoupling stage, the FLOP count of the joint detectors is two orders of magnitude higher than the decoupling-based detectors. Moreover, the suboptimal detectors implemented in the second phase of the receivers, identified as D-SVD/PBLD-LR and D-MMSE/PBLD-LR, offer significant complexity reductions compared to the optimal detectors versions, namely D-SVD/ML-W and D-MMSE/ML-W. This reduction is particularly observable in systems with few users, as the number of users is increased, the complexity of the receivers is dominated by the decoupling phase. Finally, regarding the equivalence between D-SVD/ML-W and D-ZF/ML-C, it is worthy to note that, although they present similar complexity when 4-user systems are considered, the $\mathrm{ZF}$ decoupler is preferable due to the fact that the receiver based on this decoupler presents only $22 \%$ of the complexity required by the SVD-based receiver when the system scales to 16 users.

The detection performance of the receivers is also evaluated considering propagation effects, namely antenna correlation and shadowing. For the channel correlation between neighbor-
TABLE II

COMPUTATIONAL COMPLEXITY OF THE RECEIVERS (FLOPS $\times 10^{3}$ ).

\begin{tabular}{llll}
\hline Receivers & $\mathrm{A}$ & $\mathrm{B}$ & $\mathrm{C}$ \\
\hline D-ZF/ML-C & 70.6 & 281.0 & 356.6 \\
D-SVD/ML-W & 61.5 & 689.0 & $1,593.0$ \\
D-MMSE/ML-W & 96.6 & 234.2 & 385.9 \\
D-SVD/PBLD-LR & 38.1 & 667.0 & $1,440.0$ \\
D-MMSE/PBLD-LR & 38.1 & 185.1 & 233.2 \\
PBLD-LR & $3,233.0$ & - & - \\
PBLD & $2,172.0$ & - & - \\
\hline
\end{tabular}

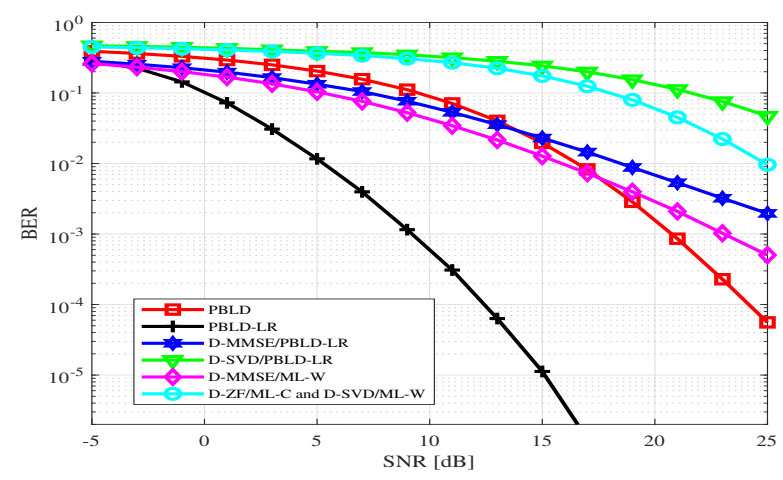

Fig. 3. Bit error rate of the receivers -4 users, $N_{R}=16$, with propagation effects: $\rho=0.8$ and $\sigma=6 \mathrm{~dB}$.

ing antennas, Kronecker model was employed. The channel matrix considering correlation effects, $\mathbf{G}_{n}$, is defined by $\mathbf{G}_{n}=\mathbf{R}(\rho)^{\frac{1}{2}} \mathbf{H}_{n} \mathbf{R}(\rho)^{\frac{1}{2}}$, where $\mathbf{R}$ pre/post-multiplying $\mathbf{H}_{n}$ represents the correlation effect between receiving and transmitting antennas, and $\rho$ is the correlation coefficient between neighboring antennas, as presented in [11]. Finally, a lognormal shadowing effect jointly with the antenna correlation, produces the resulting channel matrix $\tilde{\mathbf{G}}_{n} \in \mathbb{C}^{N_{R} \times N_{T}}$, given by $\tilde{\mathbf{G}}_{n}=\beta_{n} \mathbf{G}_{n}$, where $\beta_{n}$ is a log-normal random variable given by $\beta_{n}=10^{\frac{\sigma \mathcal{N}(0,1)}{10}}$. In turn, $\sigma$ represents the lognormal shadowing spread parameter given in decibel, and $\mathcal{N}(0,1)$ is a zero mean and unit variance Gaussian random variable. Considering these propagation effects, Fig. 3 shows that the joint detector PBLD presents a high performance loss. This is credited to the poor performance of the projection matrix filter bank and the ZF equalizer in ill-conditioned highdimension channel matrices. Additionally, MMSE-decouplerbased receivers experienced the least performance loss among all other strategies based on other decouplers, in spite of presenting low-computational complexity.

\section{CONCLusions}

A variety of receivers were presented for the uplink of multiuser GSM MIMO systems. These strategies were compared in terms of BER and computational complexity, under different system configurations and channel characteristics. Simulations presented the performance of the different decoupling strategies and also the optimal and suboptimal detectors. 


\section{REFERENCES}

[1] R. Y. Mesleh, H. Haas, S. Sinanovic, C. W. Ahn, and S. Yun, "Spatial modulation," IEEE Trans. Veh. Technol., vol. 57, no. 4, pp. 2228-2241, Jul. 2008, DOI: 10.1109/TVT.2008.912136.

[2] J. Wang, S. Jia, and J. Song, "Generalised spatial modulation system with multiple-active transmit antennas and low complexity detection scheme," IEEE Trans. Wireless Commun., vol. 11, no. 4, pp. 1605-1615, Apr. 2012, DOI: 10.1109/TWC.2012.030512.111635.

[3] J. Cal-Braz and R. Sampaio-Neto, "Low-complexity sphere decoding detector for generalized spatial modulation system," IEEE Commun. Lett., vol. 18, no. 6, pp. 949-952, Jun. 2014, DOI: 10.1109/LCOMM.2014.2320936.

[4] —, "Nested maximum likelihood group detection in generalized spatial modulation MIMO systems," IEEE Commun. Lett., vol. 18, no. 6 pp. 953-956, Jun. 2014, DOI: 10.1109/LCOMM.2014.2323252.

[5] _ , "Projection-based list detection in generalized spatial modulation MIMO systems," IEEE Commun. Lett., vol. 19, no. 7, pp. 1145-1148, Jul. 2015, DOI: 10.1109/LCOMM.2015.2435007.

[6] J. K. Kim, H. Moon, K. Hyun, and S. K. Park, "The performance of spatial modulation in uplink multi-user system," in 2014 IEEE Region 10 Conference (TENCON 2014), Bangkok, Thailand, Oct. 2014, DOI: 10.1109/TENCON.2014.7022393.

[7] W. Wang and W. Zhang, "Spatial modulation for uplink multiuser mmWave MIMO systems with hybrid structure," IEEE Trans. Commun., vol. 68, no. 1, pp. 177-190, Jan. 2020, DOI: 10.1109/TCOMM.2019.2948340.

[8] T. L. Narasimhan, P. Raviteja, and A. Chockalingam, "Generalised spatial modulation in large-scale multiuser MIMO systems," IEEE Trans. Wireless Commun., vol. 14, no. 7, pp. 3764-3779, Jul. 2015, DOI: 10.1109/TWC.2015.2411651.

[9] J. L. C. Juajinoy, J. Cal-Braz, and R. Sampaio-Neto, "Receivers for the up-link of multiuser MIMO systems with spatial modulation," in Proceedings of the XXXV Brazilian Telecommunicatios and Signal Processing Symposium (SBrT 2017), Sao Pedro, Brazil, Sep. 2017, pp. 1014-1018, DOI: $10.14209 /$ sbrt.2017.57.

[10] V. Stankovic and M. Haardt, "Generalized design of multi-user MIMO precoding matrices," IEEE Trans. Wireless Commun., vol. 7, no. 3, pp. 953-961, Mar. 2008, DOI: 10.1109/LCOMM.2008.060709.

[11] L. Arevalo, R. C. de Lamare, M. Haardt, and R. Sampaio-Neto, "Decoupled signal detection for the uplink of massive MIMO in 5G heterogeneous networks," EURASIP Journal on Wireless Communications and Networking, vol. 2017, no. 131, pp. 1-15, Jul. 2017, DOI: 10.1186/S13638-017-0916-1.

[12] D. Wubben, D. Seethaler, J. Jalden, and G. Matz, "Lattice reduction," IEEE Signal Process. Mag., vol. 28, no. 3, pp. 70-91, May 2011, DOI: 10.1109/MSP.2010.938758.

[13] R. P. David, "Personal notes email:rpdavid@inmetro.gov.br."

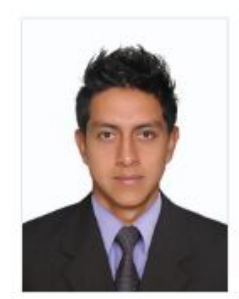

José Calpa received the Diploma degree in Electronic Engineering from Universidad de Nariño, Colombia, in 2013, and the M.Sc. degree in Electrical Engineering from Pontifícia Universidade Católica do Rio de Janeiro (PUC-Rio), Brazil, in 2017, where he is currently pursuing the Ph.D. degree in Electrical Engineering. His main research interests include communication theory, digital transmission, wireless communications systems and signal processing for communications.

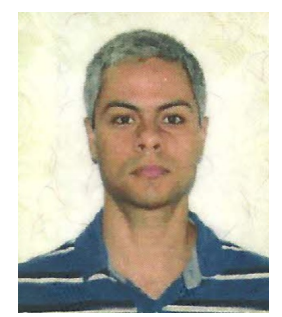

João Cal Braz received the $\mathrm{PhD}$ degree in Elecrical Engineering from Pontifical Catholic University of Rio de Janeiro (PUC-Rio), Brazil, in 2015. He is a researcher at the National Metrology Institute of Brazil (Inmetro). His main research interests include statistical signal processing, communication theory, digital transmission and mobile radio channel characterization.

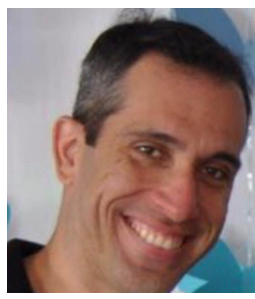

Rodrigo Pereira David Graduate in Electrical Engineering from the University of the State of the Rio de Janeiro (2002), MSc (2007) and PhD (2014) in Communication Systems from the Pontifical Catholic University of Rio de Janeiro. He is currently researcher at Laboratory of Telecommunications in the Brazilian Institute of metrology (INMETRO). Have experience in Electrical Engineering with emphasis on Telecommunication Systems, acting on the following topics: satellite communication systems, signal processing for communications, digital broadcasting and wireless communications systems.

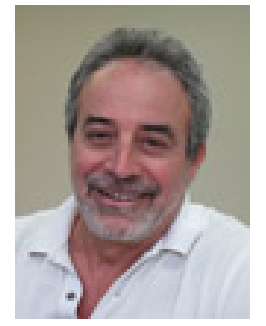

Raimundo Sampaio Neto received the Diploma and the M.Sc. degrees from Pontifícia Universidade Católica do Rio de Janeiro (PUC-Rio), Brazil in 1975 and 1979, respectively, and the Ph.D. degree from the University of Southern California (ISC), Los angeles, in 1983, all in electrical engineering. From November 1983 to June 1984 he was a PostDoctoral fellow at the Communication Sciences Institute of the Department of Electrical Engineering at USC, and a member of the technical staff of Axiomatic Corporation, Los Angeles. He is currently a researcher with the Center for Studies in Telecommunications (CETUC) and an Associate Professor of the Department of Electrical Engineering, PUC-Rio, where he has been since July 1984. In 1991, he was a Visiting Professor in the Department of Electrical Engineering at USC. He has participated in various projects and consulted for several private companies and government agencies. His areas of interest include communication theory, digital transmission and signal processing for communications, areas in which he has published more than 200 papers in refereed journal and conferences. He was a co-organizer of the Session on Recent Results for the IEEE Workshop on Information Theory, 1992, Salvador, Brazil. He also served as Technical Program Cochairman for IEEE Global Telecommunications Conference (Globecom 99) held in Rio de Janeiro and as a Technical Program Member for several national and international conferences. He was in office for three terms on the Board of Directors of the Brazilian Communications Society (SBrT). He served as a member of its Advisory Council for four terms and as Associate Editor of the society journal: Journal of Communications and Information Systems. He is currently a Emerit Member of SBrT. 